\title{
LONG-TERM THERMAL COMPENSATION OF 5-AXIS MACHINE TOOLS DUE TO THERMAL ADAPTIVE LEARNING CONTROL
}

\author{
P. Blaser ${ }^{1 *}$, J. Mayr ${ }^{2}$, K. Wegener ${ }^{1}$ \\ ${ }^{1}$ ETH Zurich, Institute of Machine Tools and Manufacturing (IWF), Zurich, Switzerland \\ 2inspire AG, Zurich, Switzerland \\ *Corresponding author; e-mail: blaser@iwf.mavt.ethz.ch
}

\begin{abstract}
This paper presents a prediction and compensation approach for thermal errors of 5-axis machine tools, based on supervised online machine learning. Process-intermittent probing is used to identify and update a thermal autoregressive with exogenous input (ARX) model. The approach is capable of predicting and compensating thermal displacements of the tool center point based on changes in the environmental temperature, load-dependent changes and boundary condition changes and states, like dry or wet machining. The self-optimized machine tool shows very stable long-term behavior under drastically varying machining and boundary conditions. The implementation is validated on a set of thermal test pieces. The test pieces show that the major share of thermal workpiece errors are reduced by the thermal adaptive learning control.
\end{abstract}

\section{Keywords:}

Thermal behavior; Compensation; Self-optimization; Machine learning

\section{INTRODUCTION}

The accuracy of 5-axis machine tools is a key factor to modern manufacturing of multi-axes machined workpieces. According to Bryan [Bryan 1990] and Mayr et al. [Mayr 2012] thermally induced deviations account for the largest single source of errors in machine tools and measurement machines. The variation of the structural temperature leads to position and orientation errors of the tool center point (TCP) relative to the workpiece. Reducing the thermally induced errors in the whole working space without increasing the energetic demand is a topic of great industrial relevance. The increasing number of publications related to thermal issues in machine tools also shows the growing academic interest. [Hernandez-Becerro 2018].

Thermal errors can have particularly significant effects on the accuracy of machine tools. Causes are thermal deformations of the machine elements caused by heat sources that exist within the structure, for example: ball screws, bearings, axis drive motors, friction on the guideway surfaces, and the flows of coolant, and external influences as ambient temperature changes.

Since the thermal behavior of machine tools is affected by a variety of factors, reducing thermal errors is a complex process. The avoidance of the occurrence of thermal errors solely by design measures of the machine structure within an acceptable economical effort is not possible.

Compensation is a process where the thermal errors present at a particular time are corrected by adjusting the position of a machine's axes by an amount equal to the errors at that position. Researchers have employed various techniques such as a finite-element method for instance by
[Mayr 2015a, Mian 2013] and finite-difference method by for example [Bossmanns 1999, Mayr 2009] in modelling the thermal characteristics. However, building a physical model can be a great challenge due to problems of establishing the boundary conditions and accurately obtaining the characteristics of heat transfer. Therefore, testing of the machine tool is still required to calibrate the model for successful application of the technique.

In contrast, other techniques use empirical modelling, where the model is based on experimental measurements of the machine tool, rather than calibrating an existing model. Over the past several decades, researchers have investigated different kinds of such strategies to establish thermal error models, as summarized in [Ramesh 2000]. The goal is to find the optimized thermal error model with high accuracy and robustness as the model determines the correctness and effectiveness of the thermal error compensation subsequently.

Ideally, the residual deviations between the predicted errors and the actual machine tool errors will approach zero. However, the predicted system behavior is always different from the physical one. Additionally, the actual machining conditions may be different from the machining conditions used to derive the empirical model, which leads to model uncertainties. Mou and Liu [Mou 1995] stated that this can lead to prediction errors especially for small batch productions, where the sequence of manufacturing processes changes frequently as then also does the direction and rate of change of thermal effects.

As stated by [Venugopal 1986] the deformation of a body at a particular time depends only on the temperature of that body at that particular instant in time. Models based on this 
assumption aim at building a correlation model between the current temperature measurements and the thermal deformations of the machine. The researchers then tried to establish a thermal prediction model based on the correlation of several temperature inputs and the thermal TCP deviations. In some publications, approaches can be found, where also multiple variables, such as the spindle speed, motor current, historical information etc. are used as inputs to model to the thermal errors, see [Brecher 2009, Gebhardt 2013, Mayr 2015b, Yang 2005] just to name a few.

According to Yang and $\mathrm{Ni}$ [Yang 2003] models that only consider the current state of the machine are considered a static approach and are widely used for machine tool thermal error modeling in industry. They also state that static models suffer from poor robustness and prediction accuracy, whereas dynamic models are superior in terms of robustness and model accuracy.

Autoregressive models are a special kind of dynamic regression models which are popular for time series data modeling [Ljung 1999]. As the thermal error varies continuously with time, researchers applied the autoregressive method for the thermal error modeling and compensation. For example, [Li 1997] found out that the thermal errors of a spindle at a certain instance depends on the thermal errors at the last instance and the spindle speed at the current instance, under the assumption of no load or finishing conditions.

Yang [Yang 2002] reviewed several thermal error compensation approaches and located major barriers, that prevent the practical implementation in real industrial production. He states that the accuracy and robustness of thermal models is insufficient and that the thermal errors have to be measured in an efficient manner during production. He especially points out, that due to statistical uncertainties, assumptions in the model and the constantly changing boundary conditions, the error models derived from pre-process calibration are not necessarily accurate enough in the long term. They need to be verified and updated iteratively as the machine tool is continually used.

In [Blaser 2017] a new approach for an adaptive learning control (ALC) for thermal error compensation of rotary axes of 5-axis machine tools has been presented. The schematic concept of the ALC is illustrated in Fig. 1. During a calibration phase, the thermal errors and the current boundary and machining conditions are measured by various sensors to obtain the first set of parameters for the thermal prediction model. The thermal errors are measured by process-intermittent probing with a touch trigger probe. After the phenomenological model is obtained and the thermal error compensation starts, the errors are measured with a reduced frequency to increase the machine productivity. The compensated thermally induced errors are compared to a defined action control limit. If the deviations exceed this threshold, a new set of model parameters is obtained with the data gathered since a specific point in time, so that the parameters of the error model can be adapted to the present situation.

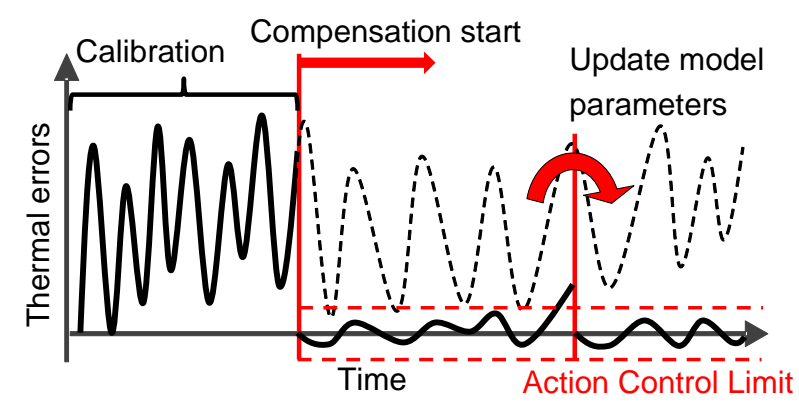

Fig. 1: Illustration of the concept of ALC for thermal error compensation, adapted from [Blaser 2017].

\section{ADAPTIVE LEARNING CONTROL (ALC) FOR THERMAL ERROR COMPENSATION}

The functionality of the ALC procedure is explained in [Blaser 2017, Blaser 2018, Mayr 2018]. However, it is briefly reviewed in this section, since it provides the basis for the research presented in this paper.

The objective of the adaptive self-learning thermal compensation is to reduce the thermally induced TCPdeviations and to improve the long-term accuracy of the machine tool in both material removal and on-machine inspections with as little as possible production interruptions. The procedure is able to adapt its model parameters to changes in the process and boundary conditions. This methodology is also able to adjust the onmachine measurement time intervals according to the predefined action control limits to ensure high productivity at a set accuracy range of the phenomenological model, as seen in Fig. 1.

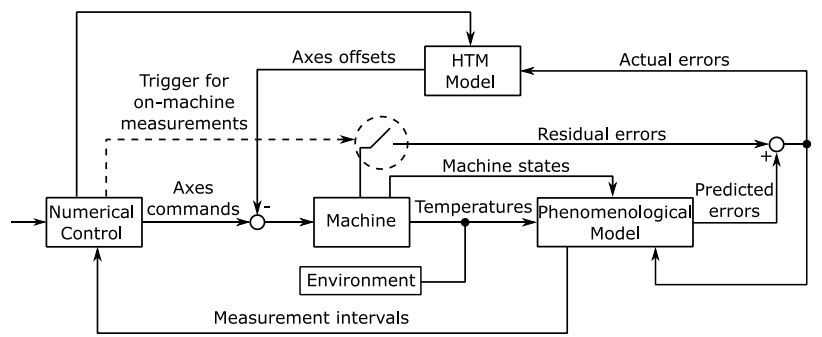

Fig. 2: Schematic diagram of ALC. The dashed line represents the trigger for on-machine measurements. The phenomenological model adaptively adjusts the measurement intervals [Blaser 2018].

Fig. 2 shows a schematic diagram of the ALC methodology. A measurement procedure is used which is capable of identifying the thermal position and orientation errors of a rotary axis with a touch trigger probe and a precision artefact mounted on the machine table. The axis error model used is based on the rigid body assumption and the use of homogeneous transformation matrices (HTM) to obtain the thermal displacements of the TCP relative to the workpiece position. The input of the HTM model are the predicted errors and the axis position of the machine tool and the output is an axes offset that shifts the axis origin in the opposite direction of the occurring thermal error. The phenomenological model predicts the thermal behavior by tracking multiple machine states as well as temperature sensor values on and around the machine structure. The extension of using machine states as input for the model was introduced by Blaser et al. [Blaser 2018] to include cutting fluid conditions. The outputs of the phenomenological model, the predicted errors, are 
compared with the on-machine measurements obtained by the touch probe at discrete points in time. This comparison is used to periodically update the parameters of the thermal error model. To adapt to changing working conditions the approach is capable of modifying the NC-Code, with the use of a numerical control interface, to adjust the time intervals between on-machine measurements. This allows to dynamically adapt the amount of measurements to tradeoff between accuracy and productivity. The onmachine measurement procedure only needs a few measurement points and allows a fast tracking of the thermal TCP-deviations. The repeatability and the measurement uncertainty of this on-machine measurement cycle is presented by Blaser et al. in [Blaser 2017, Blaser 2014].

To the fact, that the measurement intervals of the onmachine inspection can be triggered arbitrarily, the sample rate of the actual measured thermal errors is changing constantly. Mayr et al. [Mayr 2018] presented an adaptive self-learning algorithm for thermal error compensation for arbitrary sample rates. It is shown that with the extension of a weighting matrix for the least squares (LSQ) estimation of the ARX system parameters, the thermal model can handle any sampling rate coming from the arbitrary TCP measurements. The self-learning algorithm determines the optimal model order of the ARX model and monitors the compensated thermal location errors. If the errors exceed a set action control limit, a new set of parameters is automatically estimated.

The ALC approach strongly depends on the selection of optimal inputs. Only thermal influences captured by those inputs can be modeled and compensated. Furthermore only repeatable effects can be accurately mapped to the occurring thermal TCP deviations.

\section{PARAMETER STUDY OF ALC}

The quality of the ALC is highly dependent on a handful of parameters. In this section an analytical investigation of those parameters is performed. Tab. 1 shows a list of the seven most influential parameters for the thermal compensation with ALC. Over the duration of an experiment the approach can be divided in three phases, the calibration phase $(\mathrm{CP})$, the compensation phase (Post $\mathrm{CP}$ ) and the No-Good phase (NG). The CP consists of the first few hours until a first set of model parameters is computed and the compensation phase (Post CP) starts. The NG phase is triggered, when an on-machine measurement is performed and the residual errors exceed the action control limit. Then the measurement frequency is increased and a model parameter update is performed after a certain amount of measurements.

Tab. 1: Influential parameters for the thermal compensation with $A L C$ and the corresponding range for the parameter study.

\begin{tabular}{ll}
\hline Parameter & Values \\
\hline Calibration Phase (CP) & $6.5 / 12 / 16 / 24 / 36 / 48 / 60[\mathrm{~h}]$ \\
Measurement Interval (CP) & $5 \mathrm{~min}$ \\
Measurement Interval (Post CP) & $1-12 / 24 / \mathrm{Inf}[\mathrm{h}]$ \\
Measurement Interval (NG) & $5 / 10 / 15 / 20 / 25 / 30 / \operatorname{Inf}[\mathrm{min}]$ \\
Action Control Limit & $5 \mu \mathrm{m} \mathrm{resp.} 15 \mu \mathrm{m} / \mathrm{m}$ \\
Nr. of measurements in NG & $6 / 12 / 18 / 24 / \mathrm{Inf}[\mathrm{meas}$.] \\
Parameter Update Interval & $8-96 / 192[\mathrm{~h}]$ \\
\hline
\end{tabular}

For the parameter study, five out of these seven parameters are closer investigated, the ranges of the examined values are listed in Tab. 1. All possible combinations are studied, which results in a total of 2'191 virtual experiments, that need to be performed.

To test the dependability of the compensation quality on different thermal heat inputs, four experiments are performed on a real 5-axis machine tool. During the experiment three temperatures and seven position and location errors of the rotary axis are recorded. The different load cases (A-D) for the measurements are depicted in Fig. 3. To induce an alternating thermal load into the machine tool an arbitrary speed profile of the $\mathrm{C}$-axis is performed to simulate a fast changing small batch production. In measurement $D$ in addition to the arbitrary speed profile of the $\mathrm{C}$-axis, the cutting fluid supply through flexure tubes aiming at the working table is switched on and off during different lengths of time intervals.

The machine tool under investigation operates in normal workshop conditions without any environmental temperature control. The resulting temperature changes of the environment (ENV), the workspace respectively the cutting fluid (CF) and the $\mathrm{C}$-axis coolant due to the load cases specified in Fig. 3 are shown in Fig. 4. In load case $D$, instead of measuring the workspace temperature the CF temperature is measured. As one can see, the environmental temperature is fluctuating in daily cycles and is heavily influenced by the hall door right next to the machine. As shown in [Blaser 2017, Blaser 2018, Mayr 2018], the inputs as seen in Fig. 4 can be used for the ALC and yield accurate compensation results for the presented load cases.

Applying all the different combinations of parameters, see Tab. 1, on these four load cases a total of 8'764 simulation runs results. To handle the big amount of simulations and data a software environment is implemented that allows a parallelized computing and therefore a faster evaluation.

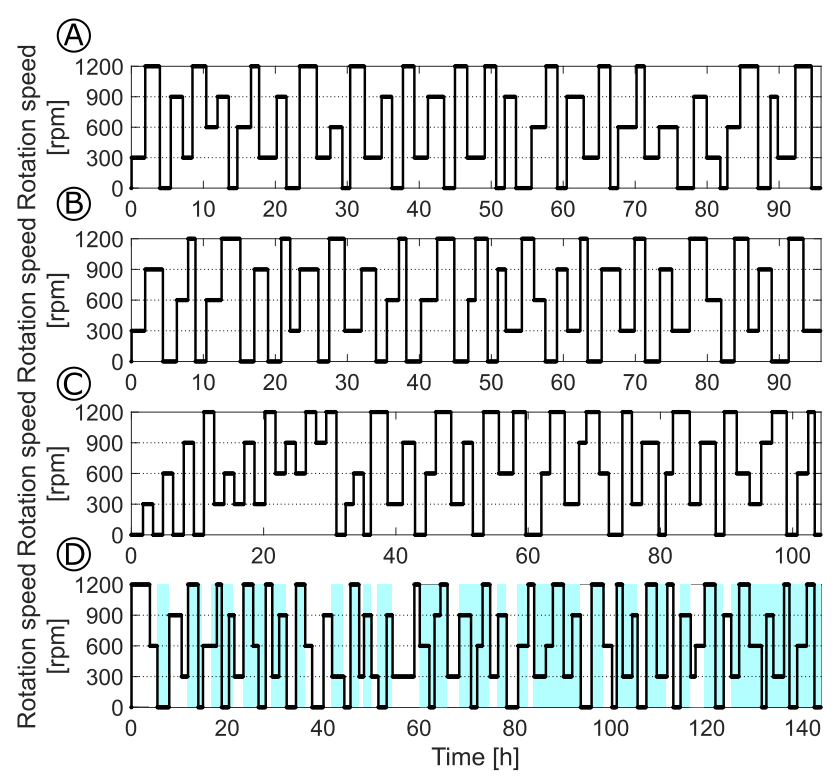

Fig. 3: Load cases with varying rotational speed of the $C$ axis and changes of cutting fluid states. The cyan areas depict the time intervals, where the cutting fluid supply is switched on. 

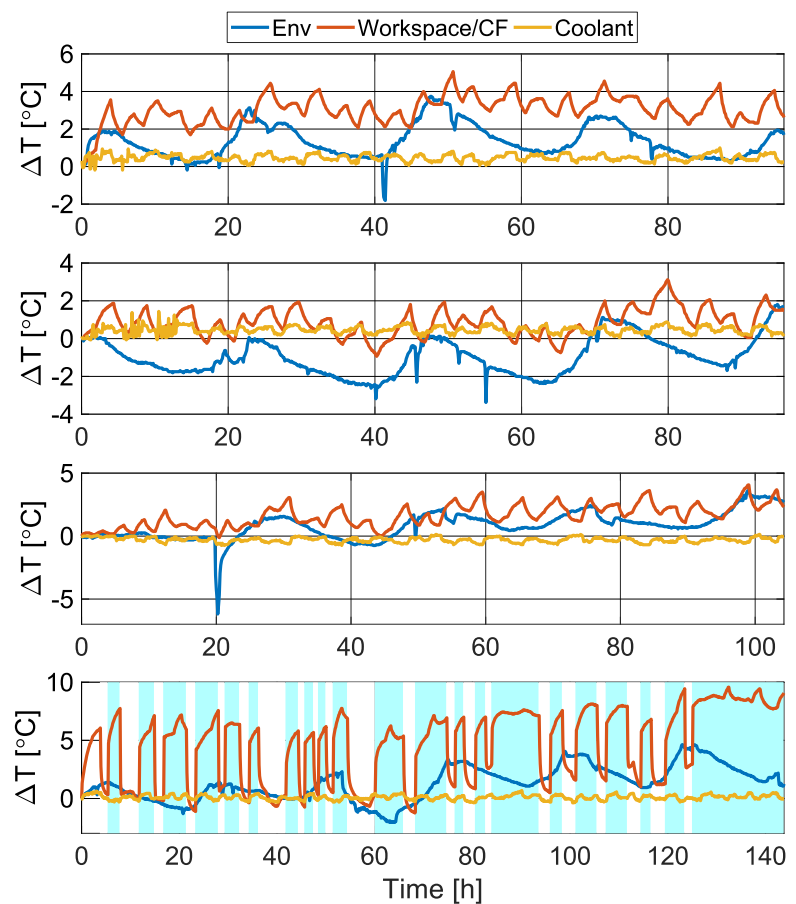

Fig. 4: Temperature changes of the environment (ENV), the workspace respectively the cutting fluid (CF) and the $C$-axis coolant due to the corresponding load cases. The cyan areas depict the time intervals, where the CF supply is switched on.

\subsection{Quality indicators}

To evaluate the goodness of fit of the thermal errors, four different indicators are used. Each variable describes the compensation quality with a different focus. In the following equations, the subsequent nomenclature is used. The measured deviation is abbreviated with $y_{t}$ and the fitted deviations $\widehat{y_{t}}, N$ depicts the number of total samples, $C P$ is the number of measurements until the start of the compensation.

The Peak value PV measures the relation between the biggest peak of the compensated to the uncompensated time series, Eq. (1). It evaluates the biggest outliers, not considered the frequency of occurrence. The smaller the PV of the residual errors, the better the compensation.

$P V=\frac{\max \left(\left|y_{t}-\widehat{y_{t}}\right|\right)}{\max \left(\left|y_{t}\right|\right)} * 100, \forall t \in[C P, N]$

The root mean square error (RMSE) is the square root of the average of squared errors, see Eq. (2) and measures the difference between samples of the measured and the predicted deviations. The RMSE is always non-negative and not limited, a value of 0 indicates a perfect fit to the data. In general, a lower RMSE is better than a higher one. The effect of each error on the RMSE is proportional to the size of the squared error; thus, larger errors have a disproportionately large effect on RMSE. Consequently, RMSE strongly penalizes higher differences in the fit from the original data.

$R M S E=\sqrt{\frac{\sum_{t=C P}^{N}\left(\widehat{y_{t}}-y_{t}\right)^{2}}{N-C P}}$

A percentile is a measure indicating the value below which a given percentage of observations in a group of observations falls. For example, the 20th percentile (P20) is the value below which $20 \%$ of the observations may be found. In this work, the 99th percentile (P99) is used to describe the residuals according to Eq. (3). To calculate the percentile $\mathrm{Pr}$ the deviations need to be sorted from lowest to highest. In Eq. (3) $v$ corresponds to the absolute residuals $\left|\widehat{y_{t}}-y_{t}\right|$ and $\lfloor x\rfloor$ stands for the floor function, whereas $(x \% 1)$ uses the modulus to represent the remainder after division by 1 . Eq. (4) shows the derivation of the sample index for the $P^{\text {th }}$ percentile. The advantage of the percentile is the retaining of the physical basis, so one can directly evaluate the remaining deviation of the residuals.

$$
\begin{aligned}
& \operatorname{Pr}(v)=v_{\lfloor x\rfloor}+(x \% 1)\left(v_{\lfloor x\rfloor+1}-v_{\lfloor x\rfloor}\right), \forall x \in[C P, N] \\
& x(P)=\left\{\begin{array}{c}
N \cdot P+\frac{1}{2}, \forall p \in\left[p_{1}, p_{N-C P}\right] \\
1, \forall p \in\left[0, p_{1}\right] \\
N, \forall p \in\left[p_{N-C P}, 1\right]
\end{array} \forall P \in[0,1]\right.
\end{aligned}
$$

with:

$p_{i}=\frac{1}{N-C P}\left(i-\frac{1}{2}\right), i \in[1, N-C P]$

The symmetric mean absolute percentage error (SMAPE) is an accuracy measure based on percentage errors. The absolute difference between $y_{t}$ and $\widehat{y}_{t}$ is divided by the sum of absolute values of the actual value $y_{t}$ and the predicted value $\widehat{y_{t}}$. The value of this calculation is summed for every fitted point $t$ and divided again by the number of fitted values $N-C P$, see Eq. (6). SMAPE has both a lower bound and an upper bound, so it provides a result between $0 \%$ and $100 \%$. A limitation of SMAPE is that if the actual value or forecast value is 0 , the value will hit the upper limit, even though the difference between the data and the fit could have been small.

$S M A P E=\frac{100}{N-C P} \sum_{t=C P}^{N} \frac{\left|\widehat{y_{t}}-y_{t}\right|}{\left|\widehat{y_{t}}\right|+\left|y_{t}\right|}$

\subsection{Results of parameter study}

The axis under investigation is the rotary axis $C$ of a 5 -axis machine tool, this results in seven time-varying thermal errors, Exoc, EYOC, $E_{Z 0 T}, E_{R O T}, E_{A 0 C}, E_{B O C}$ and $E_{\mathrm{COC}}$. For every thermal error the quality indicator can be evaluated. In combination with all the varied parameters, this results in too many degrees of freedom for visual inspection in a single plot. Nevertheless, in this section, a sequential analysis of the different influences is shown and an optimal set of parameters found.

To reduce the amount of free parameters an additional quality indicator is introduced, the productivity $P d$.

$P d=\left(1-\frac{M * T_{M}}{T_{\text {total }}}\right) * 100$

In Eq. (7) $T_{M}$ stands for the measurement time (95 seconds) of a single on-machine measurement and $T_{\text {total }}$ for the total duration of the experiment. $M$ corresponds to the total amounts of measurements performed. All the parameters listed in Tab. 1. are directly influencing the amount of measurements $M$. The $\mathrm{Pd}$ shown here is no real measure of machine productivity, since a $100 \%$ availability is assumed. In reality the on-machine measurements can also be performed during non-productive times, as for example shift changes, operator breaks, between setups, etc.

To analyze the influence of the length of the calibration phase on the SMAPE a boxplot is shown in Fig. 5. The figure shows the average SMAPE of all seven time-varying thermal errors. The boxplot consists of the median, the $25^{\text {th }}$ respectively $75^{\text {th }}$ quantile as well as outliers above and below the quantiles. It can be seen that the CP has an influence on the SMAPE and especially on the outliers. Nevertheless, the impact on the median value is not so significant. It is also visible, that the SMAPE values differ MM Science Journal | 2019 | Special Issue on HSM2019 
from load case to load case, so that not the same quality of fit can be expected for different thermal load cases of the machine tool. Especially the load case of changing cutting fluid intervals shows, that this drastic change of boundary conditions is harder to compensate for. It can be concluded, that the CP itself is not the decisive factor for the quality of the fit.

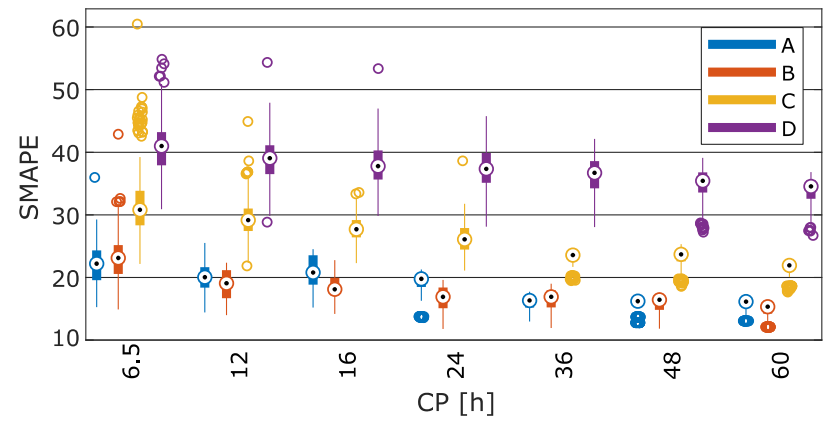

Fig. 5: Boxplot of averaged SMAPE of the thermal deviations for all load cases versus calibration phase (CP).

Fig. 6 shows that with increasing Pd the variation of the P99 is growing exponentially. A higher Pd corresponds in fewer measurements over time and thereby fewer information for the ALC. It is also visible, that there is a remarkable difference in the four experiments and that with the right combination of parameters low residual error and a high $\mathrm{Pd}$ can be achieved. As a comparison, if every 5 minutes a measurement is performed the Pd would decrease to $77 \%$. In the area of $96 \%-99 \%$, the variance of the P99 permits a statistical significant conclusion. Therefore, an adaptive measurement interval selection is needed, that increases the $\mathrm{Pd}$ if features are machined with smaller accuracy requirements and the frequency of measurements is increased, if high precision features are machined. This measure can drastically increase the overall $\mathrm{Pd}$, especially in high volume productions.

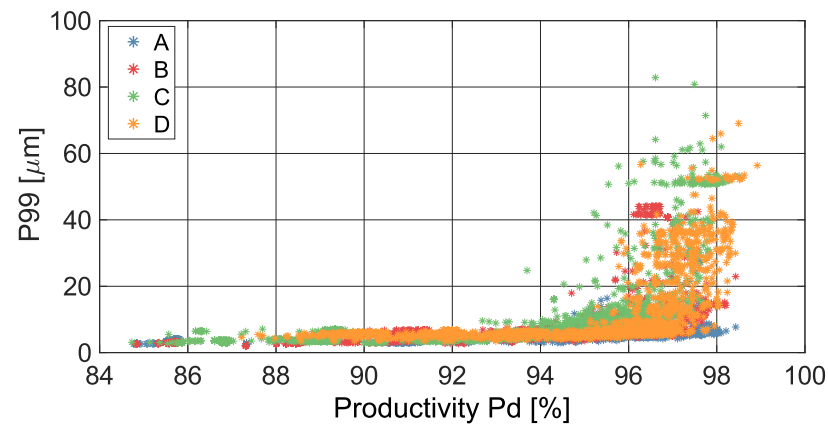

Fig. 6: The $99^{\text {th }}$ percentile of the mean of the linear thermal deformations for the different load cases $(A-D)$ versus the productivity $P d$.

The further investigation of the free parameters shows that the influence of the No Good (NG) phase is of minor importance. Whereas the frequency of on-machine measurement after the CP seem to affect the quality in a dominant way. Fig. 7 shows the influence of the on-machine measurement frequency after the CP on the RMSE. It can be seen that with more frequent measurements, the variability of the RMSE is reduced. Especially if after the CP no additional measurements are performed, labeled as "Inf" in Fig. 7, the compensation quality decreases distinctively and depends mainly on the length of the CP.

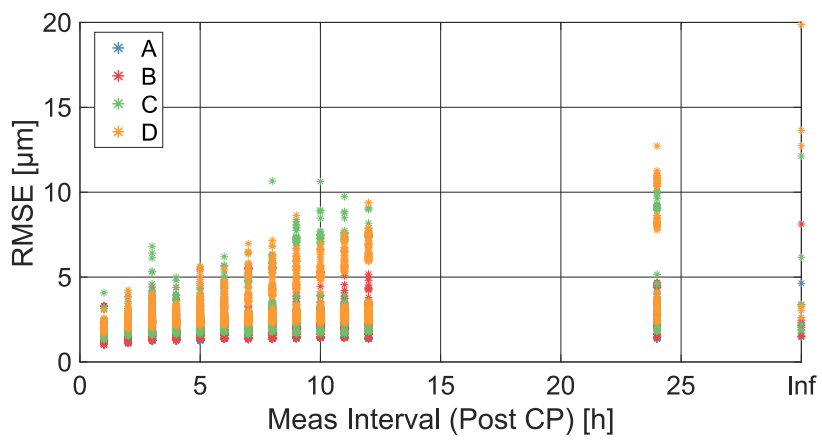

Fig. 7: RMSE of the mean of the thermal deformations of the different load cases $(A-D)$ versus the measurement interval length.

\subsection{Optimal parameter estimation}

The parameter study showed that especially the quality indicator P99 is very useful since it still contains a physical relation and one can directly relate the corresponding impact on a machined workpiece. Additionally, the reduction of the free parameters to the indicator $\mathrm{Pd}$ can simplify the problem and provides a closer industrial indicator for the trade-off. To estimate an optimal set of parameters for future compensations the following criteria is therefore introduced:

$P 99_{\text {lin }}<5 \mu m A N D \max (P d)$

In other words, Eq. (8) states, that the optimal set of parameters results in a fit, where $99 \%$ of the compensated linear errors are below $5 \mu \mathrm{m}$ and of those parameters the one with the highest $\mathrm{Pd}$ is chosen.

The optimal sets of parameters evaluated according to Eq. (8) are listed in Tab. 2. For all load cases a different set of parameters can be found, that result in a Pd of more than $95.8 \%$. It is notable; that in three cases the minimal investigated CP of 6.5 hours is sufficient, only in the case of the alternating cutting fluid supply a longer calibration phase seems preferable.

Tab. 2: Set of optimal parameters for the load cases $A-D$, from top to bottom.

\begin{tabular}{cccccc}
\hline $\begin{array}{c}\text { CP } \\
{[\mathrm{h}]}\end{array}$ & $\begin{array}{c}\text { Interval } \\
{[\mathrm{h}]}\end{array}$ & $\begin{array}{c}\text { NG Meas. } \\
{[-]}\end{array}$ & $\begin{array}{c}\text { NG Interval } \\
{[\mathrm{min}]}\end{array}$ & $\begin{array}{c}\text { P99 } \\
{[\mu \mathrm{m}]}\end{array}$ & $\begin{array}{c}\text { Pd } \\
{[\%]}\end{array}$ \\
\hline 6.5 & 3 & 6 & 25 & 4.77 & 97.25 \\
6.5 & 4 & 12 & 30 & 4.98 & 96.99 \\
$\mathbf{6 . 5}$ & $\mathbf{2}$ & $\mathbf{1 2}$ & $\mathbf{1 0}$ & 4.91 & 95.87 \\
16 & 6 & 6 & 20 & 4.98 & 96.70 \\
\hline
\end{tabular}

To find an optimal set of parameters for all four load cases, the sets listed in Tab. 2 are tested for the remaining cases. The optimal set was chosen according to the smallest P99. The best set of parameters for all load cases is found to be the set of load case $C$. So the optimal compensation starts after a calibration phase of 6.5 hours. Afterwards the measurement interval is increased from 5 minutes to 2 hours. If the set threshold is exceeded a measurement will be performed every 10 minutes for the next 2 hours, followed by a model update. 
Tab. 3: Average quality indicators of all the thermal deviations for the optimally chosen parameter set evaluated for load case $C$.

\begin{tabular}{cccccc}
\hline $\begin{array}{c}\text { Load } \\
\text { Case }\end{array}$ & $\begin{array}{c}\text { SMAPE } \\
{[\%]}\end{array}$ & $\begin{array}{c}\text { RMSE } \\
{[\mu \mathrm{m}]}\end{array}$ & $\begin{array}{c}\text { PV } \\
{[\%]}\end{array}$ & $\begin{array}{c}\text { P99 } \\
{[\mu \mathrm{m}]}\end{array}$ & $\begin{array}{c}\text { Pd } \\
{[\%]}\end{array}$ \\
\hline A & 18.37 & 1.57 & 54.79 & 4.46 & 96.42 \\
B & 18.41 & 1.90 & 63.08 & 4.62 & 96.17 \\
C & 23.88 & 1.85 & 70.58 & 4.91 & 95.87 \\
D & 34.95 & 2.42 & 69.09 & 5.29 & 96.29 \\
\hline
\end{tabular}

The corresponding quality indicator for the load cases A-D are listed in Tab. 3. It can be seen, that the P99 exceeds the defined $5 \mu \mathrm{m}$ only for the load case $\mathrm{D}$. The drawback is, that the quality indicator PV shows, that the fit suffers from few outliers and can therefore reduce the peaks only by $30 \%-45 \%$.

To verify the chosen set of parameters a simulation of the thermal error Eyoc for the load case $A$ is performed. The result is shown in Fig. 8. For comparison the thermal deviations with and without compensation is depicted. As expected the action control limit of $5 \mu \mathrm{m}$ is rarely surpassed and the compensation is very robust over the whole duration of the experiment. It can also be seen, that the compensation quality is increasing over the duration of the experiment and that the magnitude and frequency of the appearance of peaks is drastically reduced.

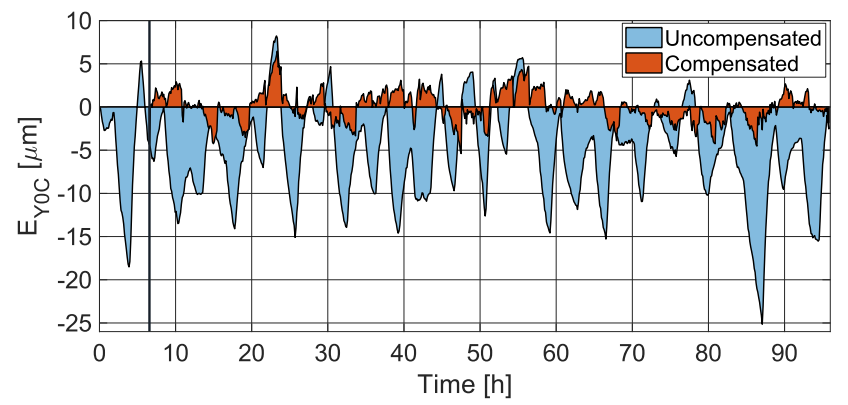

Fig. 8: Simulation of Eyoc of load case A with optimal set of parameters. CP: 6.5 h, Interval: 2 h, NG-Meas: 12 , NG-Interval: $10 \mathrm{~min}$.

To show another example and to elaborate more on the effect of increased compensation quality over time the thermal error Eyoc for load case $\mathrm{C}$ is shown in Fig. 9. It can be seen also in this load case the prediction quality of the thermal error is clearly increasing over time.

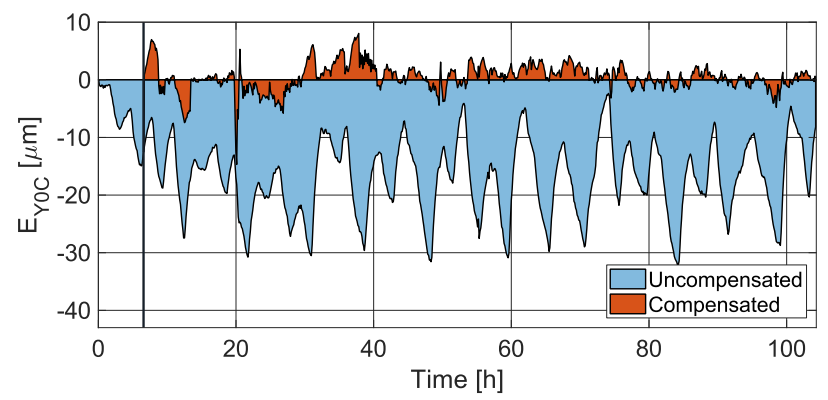

Fig. 9: Simulation of EYoc of load case $C$ with optimal set of parameters. CP: 6.5 h, Interval: 2 h, NG-Meas: 12 , NG-Interval: $10 \mathrm{~min}$.

\section{THERMAL TEST PIECE}

The thermal test piece, shown in Fig. 10 and described in detail by Wiessner et al. [Wiessner 2018a], can determine thermal errors of a machine tool in X-, Y-, Z- and either Aor B-direction. Therefore it has nine functional surfaces orthogonal to $Z$ to determine the deviations in Z-direction and the $A$ respectively $B$ rotational errors. For the $X$ - and $Y$ deviations as well as the thermal material expansion error of the test piece itself, 36 facets are milled on the outer cylindrical face with a nominal distance of $97.5 \mathrm{~mm}$ to the center. Two parallel and opposed facets are needed to measure the errors in the different directions. This enables the evaluation of nine deviations in two axes directions. One of the nine deviations in each direction is used as reference which leads to eight available points in time which is in conformity to a four hours warm-up and cool-down cycle, in accordance to ISO 230-3 [ISO 230-3:2007]. The test piece is pre-milled to keep the cutting forces as low as possible. During the test cycle the functional facets and surfaces are milled by a sequential movement of one axis at a time to avoid interpolation errors. To mill the facets the test piece is positioned by a movement of the $\mathrm{C}$ axis. The thermal test piece is fixed on the machine tool table by three M10 cap screws supported with precision washers between the table and the test piece.

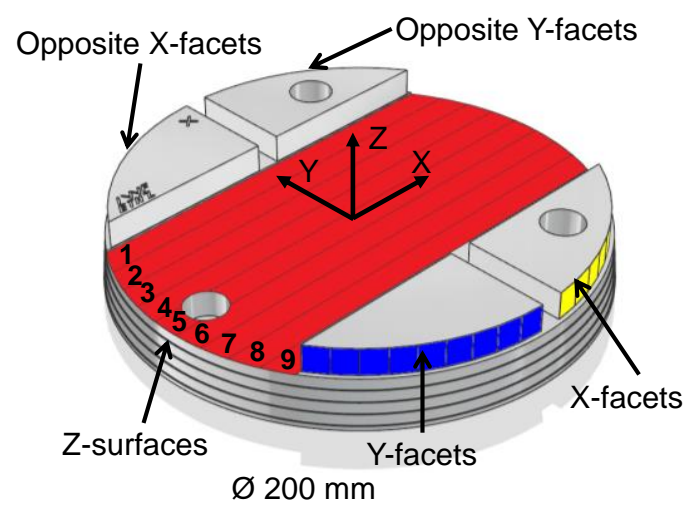

Fig. 10: Thermal test piece with highlighted functional surfaces according to Wiessner et al. [Wiessner 2018a].

\subsection{Results of ALC compensation}

To show the capability of the ALC compensation a thermal test piece with and without ALC is manufactured. The thermal load case for both test pieces is chosen according to Wiessner et al. [Wiessner 2018a]. A 4 hour warm up of the C-axis by rotating with 1'200 rpm, is followed by a 4 hour cool down phase. Once every hour five measurement surfaces are machined. Since the machine is located in a non-controlled environment, the ambient conditions for both experiments are slightly different, as shown in Fig. 12.

Following the cool down phase the thermal test pieces are measured directly on the machine. As shown by Wiessner et al. [Wiessner 2018b] on-machine measurements performed with a touch trigger probe are comparable to the coordinate measurement machine (CMM) results, since the measurements are relative in nature and therefore only influenced by the temperature change during the onmachine measurement of the specific feature. The measurement of one set of features takes approximately 2 minutes. A constant temperature can be assumed during the measurement time. The resulting thermal deviations measured on the test pieces are shown in Fig. 11. 
a)

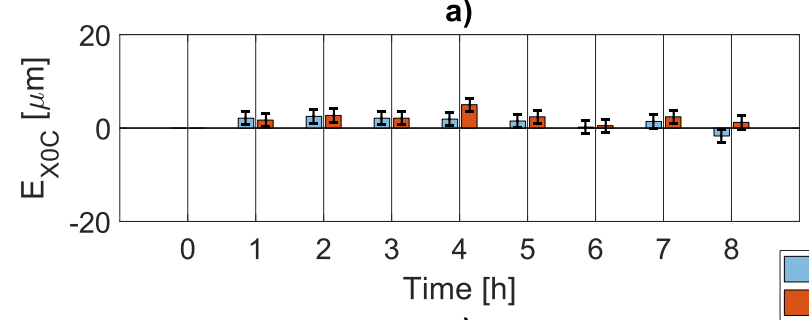

c)

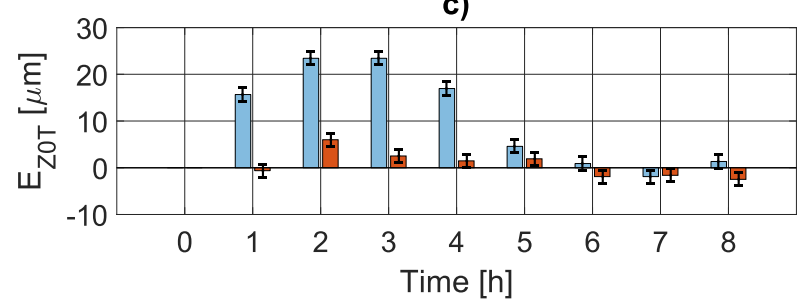

b)

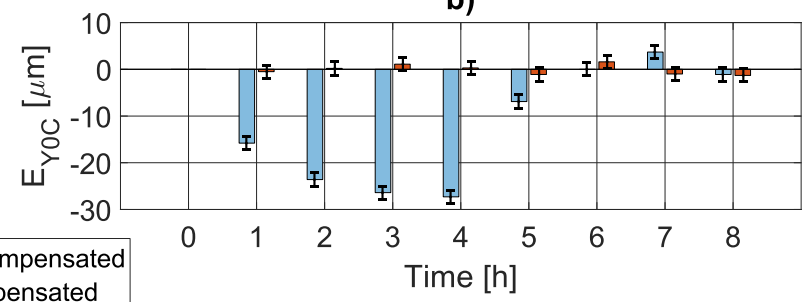

d)

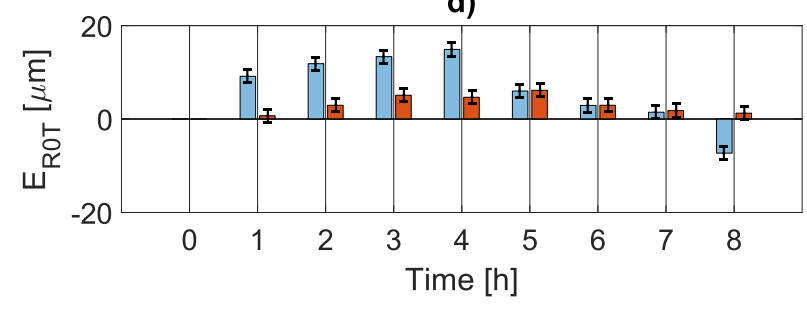

Fig. 11: Evaluation of dominant thermal errors of rotary axis $C$ with and without ALC compensation shown on a thermal test piece. The error bars illustrate the measurement uncertainty of the test piece.

The thermal prediction model for the compensated test piece was trained on load case $C$ with the optimal parameter set found in Section 3. During the manufacturing of the test piece with ALC, after every measurement surface is machined, an on-machine measurement of the precision sphere is performed, to keep the prediction model up-todate. The results in Fig. 11 show clearly, that with the ALC the thermal errors of the test piece can be significantly reduced. The biggest remaining errors are $E_{z O T}$ and $E_{R O T}$ (Fig. $11 \mathrm{c}$ and d), this can be explained by the fact, that the thermal ALC is trained to compensate the relative deviations of the machine tool table to the TCP. Not taken into account is the workpiece. In the case of the thermal test piece, which consists of aluminium and has approximately double the thermal expansion coefficient as cast iron, the influence is not negligible. The aforementioned errors are directly influenced by the thermal growth of the workpiece and therefore only partially compensated. In the directions not affected by growth of the workpiece, the errors can be kept close to zero and in the range of the measurement uncertainty.

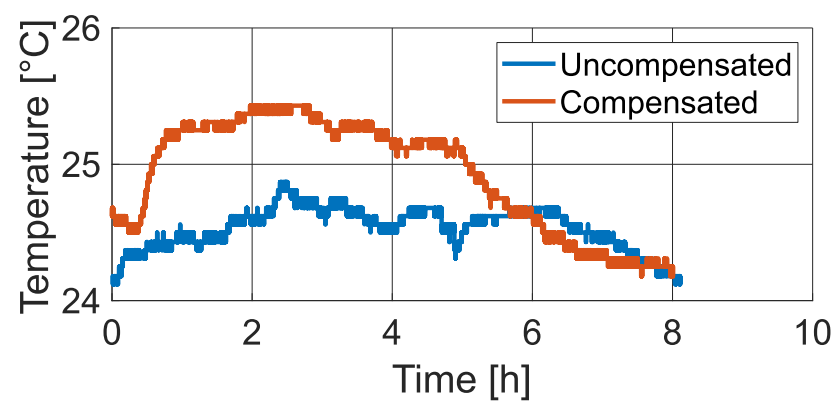

Fig. 12: Environmental temperatures during the manufacturing of the test pieces with and without ALC compensation.

\section{SUMMARY}

This paper presents an adaptive learning control for thermal error compensation for 5-axis machine tools, which is tested on four different load cases. A parameter study is performed to investigate the influence of certain control parameters for different quality indicators of the compensation. It is shown that the initial training time of the model does not have a significant impact on the compensation result, but reduces the $\mathrm{Pd}$ drastically. Whereas the measurement interval after the calibration phase is of higher importance. The study also shows, that the evaluation of the compensation quality with the mean of percentiles is very beneficial, since the physical relationship remains and can be easily interpreted by machine tool builders and operators.

With respect to Pd and the accuracy indicator P99, a set of optimal parameters is found, that shows a long-term stable compensation under different varying load cases and boundary conditions. It is shown, that even under harsh conditions like arbitrary use of cutting fluid and sudden environmental temperature changes, the ALC compensation remains stable and in the set boundaries of less than $5 \mu \mathrm{m}$ respectively $15 \mu \mathrm{m} / \mathrm{m}$.

To show the applicability of the ALC approach two thermal test pieces are machined, one with and one without ALC compensation. The test piece produced with ALC shows a drastic reduction of the occurring thermal workpiece errors, especially in the most dominant directions $Y$ and $Z$.

The ALC approach is capable of reducing the major share of thermal machine tool errors, nevertheless the experiments of the test pieces showed, that the compensation needs to be extended by the influence of the thermal deviations of the workpiece, since the workpiece can have a significant different thermal expansion than the machine tool. This can be realized by including the workpiece material expansion coefficient as an additional parameter to the ALC. However, to volumetrically compensate for workpiece errors, the actual geometry of the workpiece at the current point in time needs to be known.

To extend this approach to all five axes and the spindle, onmachine measurement methods have to be applied or developed, that allow a fast and precise estimation of the occurring thermal errors at the TCP during production.

\section{ACKNOWLEDGMENTS}

The authors would like to thank the Machine Tool Technologies Research Foundation (MTTRF), the Swiss Innovation Agency (Innosuisse) and the Swiss National Science Foundation (SNSF) for their support. 


\section{REFERENCES}

[Blaser 2014] Blaser, P., et al. Automatic compensation of thermally induced errors on five-axis machine tools. In Euspen - Special Interest Group Meeting: Thermal Issues, 2014.

[Blaser 2017] Blaser, P., et al. Adaptive Learning Control for Thermal Error Compensation of 5-Axis Machine Tools. Journal of Manufacturing Systems, Vol. 44, 2017, pp. 302309.

[Blaser 2018] Blaser, P., et al. Adaptive learning control for thermal error compensation on 5-axis machine tools with sudden boundary condition changes. In Conference on Thermal Issues in Machine Tools, Proceedings 2018, pp. 329-338.

[Bossmanns 1999] Bossmanns, B., et al. A thermal model for high speed motorized spindles. International Journal of Machine Tools and Manufacture, Vol. 39, No. 9, 1999, pp. 1345-1366.

[Brecher 2009] Brecher, C., et al. Interaction of manufacturing process and machine tool. CIRP Annals Manufacturing Technology, Vol. 58, No. 2, 2009, pp. 588607.

[Bryan 1990] Bryan, J. International Status of Thermal Error Research. CIRP Annals - Manufacturing Technology, Vol. 39, No. 2, 1990, pp. 645-656.

[Gebhardt 2013] Gebhardt, M., et al. Measuring, modeling and compensating thermally caused location errors of rotary axes. The Proceedings of MTTRF Meeting, 2013, pp. 19-24.

[Hernandez-Becerro 2018] Hernandez-Becerro, P., et al. Learning Efficient Modeling and Compensation for Thermal Behavior of Machine Tools. Proceedings of MTTRF Meeting, 2018.

[ISO 230-3:2007] ISO 230-3 Test code for machine tools Part 3: Determination of thermal effects. International Organization for Standardization ISO, Geneva, Switzerland, 2007.

[Li 1997] Li, S., et al. A study of pre-compensation for thermal errors of NC machine tools. International Journal of Machine Tools and Manufacture, Vol. 37, No. 12, 1997, pp. 1715-1719.

[Ljung 1999] Ljung, L. System identification : Theory for the User. Prentice Hall PTR, 1999.

[Mayr 2009] Mayr, J. Beurteilung und Kompensation des Temperaturganges von Werkzeugmaschinen. Diss. ETH Zürich, 2009.

[Mayr 2012] Mayr, J., et al. Thermal issues in machine tools. CIRP Annals - Manufacturing Technology, Vol. 61, No. 2, 2012, pp. 771-791.
[Mayr 2015a] Mayr, J., et al. Simulation and measurement of environmental influences on machines in frequency domain. CIRP Annals - Manufacturing Technology, Vol. 64, No. 1, 2015, pp. 479-482.

[Mayr 2015b] Mayr, J., et al. Thermal error compensation of rotary axes and main spindles using cooling power as input parameter. Journal of Manufacturing Systems. Elsevier, 2015, pp. 542-549.

[Mayr 2018] Mayr, J., et al. An adaptive self-learning compensation approach for thermal errors on 5-axis machine tools handling an arbitrary set of sample rates. CIRP Annals - Manufacturing Technology, Vol. 67, No. 1, 2018, pp. 551-554.

[Mian 2013] Mian, N. S., et al. Efficient estimation by FEA of machine tool distortion due to environmental temperature perturbations. Precision Engineering, Vol. 37, No. 2, 2013, pp. 372-379.

[Mou 1995] Mou, J. and Liu, C. An Adaptive Methodology for Machine Tool Error Correction. Journal of Engineering for Industry, Vol. 117, No. 19, 1995, pp. 389-399.

[Ramesh 2000] Ramesh, R., et al. Error compensation in machine tools - a review Part II: thermal errors. International Journal of Machine Tools and Manufacture, Vol. 40, No. 9, 2000, pp. 1257-1284.

[Venugopal 1986] Venugopal, R., et al. Thermal Effects on the Accuracy of Numerically Controlled Machine Tools. CIRP Annals - Manufacturing Technology, Vol. 35, No. 1, 1986, pp. 255-258.

[Wiessner 2018a] Wiessner, Moritz, et al. Thermal test piece for 5-axis machine tools. Precision Engineering, Vol. 52, 2018, pp. 407-417.

[Wiessner 2018b] Wiessner, M, et al. Test piece for thermal investigations of 5 -axis machine tools by on-machine measurements. In Proceedings of Conference on Thermal Issues in Machine Tools, 2018, pp. 443-452.

[Yang 2002] Yang, H. Dynamic Modeling for Machine Tool Thermal Error Compensation. University of Michigan, 2002. [Yang 2003] Yang, H. and Ni, J. Dynamic Modeling for Machine Tool Thermal Error Compensation. Journal of Manufacturing Science and Engineering, Vol. 125, No. 2, 2003, pp. 245-254.

[Yang 2005] Yang, H. and Ni, J. Adaptive model estimation of machine-tool thermal errors based on recursive dynamic modeling strategy. International Journal of Machine Tools and Manufacture, Vol. 45, No. 1, 2005, pp. 1-11. 